\title{
Vorsicht bei Valproat für Frauen
}

\section{Strengthened Warnings on the Use of Valproate in Women}

Autoren

Institute

\section{E. Elger ${ }^{1}$, T. Bast ${ }^{2}$, D. Schmidt ${ }^{3}$}

${ }^{1}$ Klinik für Epileptologie, Universität Bonn

${ }^{2}$ Epilepsiezentrum Kork, Kehl-Kork

${ }^{3}$ Arbeitsgruppe Epilepsieforschung Berlin

\author{
Schlüsselwörter \\ - Valproat-Warnung \\ - Teratogenität \\ - Entwicklungsstörung \\ Keywords \\ valproate warning \\ - teratogenicity \\ - developmental problems
}

\section{Bibliografie}

DOI http://dx.doi.org/ 10.1055/s-0034-1387540

Akt Neurol 2015; 42: 8-10

(C) Georg Thieme Verlag KG

Stuttgart · New York

ISSN 0302-4350

Korrespondenzadresse

Prof. Dr. Dieter Schmidt

Arbeitsgruppe

Epilepsieforschung Berlin

Goethestr. 5

14163 Berlin

dbschmidt@t-online.de

\section{Zusammenfassung}

$\nabla$

Die Europäische Zulassungsbehörde (EMA) und die Arzneimittelkommission der deutschen Ärzteschaft haben kürzlich die Warnung verschärft, Valproat bei Jugendlichen und Frauen, die schwanger sind oder schwanger werden können, nur dann einzusetzen, wenn andere Medikamente unwirksam sind oder nicht vertragen werden. Die Warnung wird damit begründet, dass die pränatale Valproat-Exposition das Risiko von schwerwiegenden Organfehlbildungen, hauptsächlich Neuralrohrdefekten, erhöht und bei etwa $30-40 \%$ der Kinder zu schwerwiegenden Entwicklungsstörungen führt. Diese verschärfte Warnung hat erhebliche Konsequenzen für die Beratung und den Einsatz von Valproat bei Frauen, die kurz dargestellt werden.

Die Europäische Zulassungsbehörde (EMA) und die Arzneimittelkommission der deutschen Ärzteschaft haben am 21.11.2014 bzw. am 12.12.2014 empfohlen, Valproat bei Jugendlichen und Frauen, die schwanger werden können, nur dann einzusetzen, wenn andere Medikamente versagt haben [1, 2]. Grundlage dafür sind wissenschaftliche Erkenntnisse über unerwünschte Wirkungen einer Valproat-Behandlung der Mutter für das ungeborene Kind.

Diese sehr nachdrücklich ausgesprochene Empfehlung hat erhebliche Konsequenzen für die Beratung und den Einsatz von Valproat und seinen Varianten bei Frauen in der Prä-Menopause mit Epilepsie, bipolaren Störungen und Migräne.

\section{Abstract \\ $\nabla$}

The European Medicines Agency (EMA) and the Commission on drugs of the German Physicians have strengthened warnings to prescribe valproate only in adolescents and women who can become pregnant or are pregnant when other drugs are ineffective or not tolerated. The warning is due to the risk of malformations, mainly neural tube defects, and serious developmental problems in $30-40 \%$ of babies who are exposed to valproate in the womb. The strengenthend warning has substantial consequences for counseling and the use of valproate in women which are briefly discussed.

\section{Unerwünschte Wirkungen von Valproat auf das ungeborene Kind}

Die Einnahme von Valproat im ersten Drittel der Schwangerschaft ist für Frauen mit idiopathischer (genetischer) generalisierter Epilepsie (IGE) und unklassifizierbarer Epilepsie das wirksamste, aber nicht das einzig wirksame Medikament [3]. Pränatale Valproat-Exposition erhöht allerdings deutlicher als die mit anderen untersuchten Antiepileptika das Risiko von schwerwiegenden Organfehlbildungen, vor allem Neuralrohrdefekten wie Spina bifida, Lippen-Kiefer-Gaumenspalte, Hypospadie und Herzfehler sowie von z.T. schwerwiegenden Entwicklungsstörungen inklusive Autismus und Erkrankungen des Autismusspektrums [4]. 


\section{Kindliche Fehlbildungen unter pränataler Valproat- Exposition \\ $\nabla$}

Werden in der Bevölkerung Neugeborene durch einen Kinderarzt untersucht, so wird bei 6,9\% der Kinder eine gesundheitsrelevante (große) Fehlbildung gefunden. Registriert man nur diejenigen Kinder, bei denen eine solche Fehlbildung durch Ärzte an ein Melderegister gemeldet wird, so betrifft dies 2,4\% der Kinder in der Allgemeinbevölkerung [5, 6]. Bei Frauen mit Epilepsie, die keine Antiepileptika einnehmen, liegt die Rate von kindlichen Fehlbildungen bei 3,5\% [7]. Diese Zahlen werden als Richtwert benutzt, um das Risiko für Fehlbildungen durch Medikamente abzuschätzen. Das Risiko kindlicher Fehlbildungen durch Antiepileptika wird durch die Registrierung von Neugeborenen in Schwangerschaftsregistern erfasst. Diese Register werden von nationalen oder internationalen medizinischen Fachgesellschaften geführt. Datenanalysen erfolgen dabei vorrangig für Kinder, deren Mutter mit nur einem Antiepileptikum in der Schwangerschaft behandelt wurde. Neben dem Medikament selbst analysiert man auch die Tagesdosis.

- Unter Valproat ist das Fehlbildungsrisiko dosisabhängig erhöht. So beträgt das Fehlbildungsrisiko im englischen Schwangerschaftsregister bei der Einnahme von Valproat als alleinigem Antiepileptikum 6,7\% und staffelt sich je nach Tagesdosis: 5,0\% (bis $600 \mathrm{mg}$ ), 6,1\% (bis $1000 \mathrm{mg}$ ) und 10,4\% (über 1000 $\mathrm{mg}$ [ [8].

- Das Fehlbildungsrisiko steigt weiter an, wenn Valproat mit anderen Antiepileptika kombiniert wird, insbesondere mit Lamotrigin.

- Die häufigsten Fehlbildungen durch Valproat sind eine Spina bifida, eine Lippen-Kiefer-Gaumenspalte, Herzfehler und Hypospadie [8].

\section{Kindliche Entwicklungsstörungen unter pränataler Valproatexposition}

Eine Expertengruppe hat 2014 alle verfügbaren Daten gesichtet und dabei 28 relevante Studien erfasst und ausgewertet [9]. Die Autoren fanden:

- Kinder mit pränataler Valproat-Exposition entwickelten sich in Bezug auf Laufen, Sprechen, Gedächtnis weniger gut als Kinder von Müttern mit Epilepsie ohne Antiepileptika-Einnahme (niedrigerer Entwicklungsquotient) und wiesen einen niedrigeren Intelligenzquotienten auf. Der mittlere Intelligenzquotient von pränatal Valproat-exponierten Kindern lag unter dem von Kindern epileptischer Mütter ohne Antiepileptika-Einnahme in der Schwangerschaft. Der Entwicklungsquotient von pränatal Valproat-exponierten Kindern war niedriger als derjenige von Kindern epileptischer Mütter mit Carbamazepin-Einnahme in der Schwangerschaft. Der Intelligenzquotient von pränatal Valproat-exponierten Kindern war niedriger als derjenige von Kindern epileptischer Mütter mit Lamotrigin-Einnahme in der Schwangerschaft.

- In 6 Studien wurde gezeigt, dass der negative Effekt auf das Kind abhängig von der Tagesdosis von Valproat ist. Ein erhöhtes Risiko bestand je nach Studie bei Dosierungen über 800 oder $1000 \mathrm{mg}$ pro Tag $[9,10]$.

- Zudem ist das Risiko von Autismus und Erkrankungen des Autismusspektrums bei pränataler Valproat-Exposition leicht erhöht $[4,11]$.

\section{Die Empfehlung der Gesundheitsbehörden der Euro- päischen Union und der Arzneimittelkommission der deutschen Ärzteschaft \\ $\nabla$}

Die Gesundheitsbehörden der Europäischen Union haben aus diesen Erkenntnissen Konsequenzen gezogen. Sie rechnen damit, dass bei $30-40 \%$ der Vorschulkinder von Frauen, die in der Schwangerschaft Valproat eingenommen hatten, Entwicklungsstörungen bestehen können. Die Gesundheitsbehörden der Europäischen Union und die Arzneimittelkommission der deutschen Ärzteschaft, siehe auch den Rote-Hand-Brief [2], empfehlen, dass die Behandlung mit Valproat von einem Arzt eingeleitet und überwacht werden muss, der in der Behandlung von Epilepsie, Migräne oder bipolaren Störungen Erfahrung hat. Bei der ersten Verordnung, bei Routineüberprüfungen der Behandlung, wenn ein Mädchen in die Pubertät kommt und wenn eine Frau eine Schwangerschaft plant oder schwanger wird, ist der Nutzen einer Behandlung sorgfältig gegen die Risiken abzuwägen. Patientinnen sind über die Risiken einer Behandlung und über die Anwendung einer wirksamen Verhütung aufzuklären. Sie empfehlen Valproat bei Frauen, die schwanger werden können, nur dann einzusetzen, wenn sich andere Behandlungen als unzureichend wirksam erwiesen haben oder nicht vertragen wurden.

\section{Wege aus dem Dilemma}

Es gibt unserer Ansicht nach 2 Wege aus dem Dilemma.

1. Man vermeidet Valproat generell bei Frauen. Die Aufklärung über die Risiken der Behandlung und der Nichtbehandlung mit Valproat wird dokumentiert. Der Nachteil dieser Strategie ist, dass man ihnen das nach klinischen Beobachtungen und Studien wirksamste Medikament zur Behandlung von IGE vorenthält. Ein Absetzen von Valproat und eine Nichtbehandlung eines Patienten ist wegen des erhöhten Anfallsrisikos nicht leitliniengerecht [12]. Eine Umstellung auf ein anderes Antiepileptikum birgt ebenfalls Risiken, wenn das Medikament weniger wirksam ist als Valproat und kann auch zu spät sein, wenn bereits eine Schwangerschaft besteht. Zudem erfolgt eine Umstellung von Valproat auf ein anderes Antiepileptikum typischerweise allmählich. Daher wird zumindest vorübergehend eine Mehrfachtherapie eingeführt, die ihrerseits das Fehlbildungsrisiko erhöhen kann [12]. Andere, in der Wirksamkeit zur Behandlung von IGE entweder nicht zugelassene, mit Valproat nicht mit ausreichender Evidenz verglichene oder nachgewiesen weniger wirksame zugelassene Antiepileptika für IGE sind Topiramat oder Levetiracetam (als Zusatztherapie), Lamotrigin und Ethosuximid [12]. Clobazam ist für IGE nicht zugelassen. Die Verordnung nicht zugelassener Antiepileptika ist unter Umständen nicht leitliniengerecht. Zur Behandlung anderer Epilepsien ist Valproat meist vermeidbar, weil wirksame Antiepileptika der ersten Wahl zur Verfügung stehen wie u.a. Levetiracetam oder Carbamazepin [12]. Lamotrigin ist wegen abfallender Serumkonzentrationen während der Schwangerschaft schwieriger zu handhaben als Levetiracetam [12], obwohl auch die Serumkonzentration von Levetiracetam in der Schwangerschaft abfallen kann [13]. Die Sorge um das ungeborene Kind und/oder die pharmakokinetischen Probleme in der Schwangerschaft führen wahrscheinlich bei Frauen zu Problemen mit der Compliance und können, insbesondere bei Lamotrigin, eine erhöhte Anfallsfrequenz zur Folge haben. Eine britische Studie konnte eine 
deutliche Erhöhung der SUDEP-Häufigkeit in der Schwangerschaft nachweisen; viele dieser Patientinnen erhielten Lamotrigin in Monotherapie [14].

2. Gibt es nach klinischer Erfahrung und fachärztlicher Beratung keine ähnlich wirksamen anderen Antiepileptika, verordnet man Valproat unter vier Kautelen: Die Aufklärung über die Risiken der Behandlung und der Nichtbehandlung mit Valproat wird dokumentiert. Eine effektive Verhütung der Schwangerschaft, eine Tagesdosis unter $800 \mathrm{mg}$ (s. o.) und eine Folsäuregabe schon vor der Konzeption und während der Schwangerschaft sollen sichergestellt werden. Bekannt ist, dass ein Mangel an Folsäure das Risiko von Spina bifida und Lippen-KieferGaumenspalte erhöht. Gesundheitsbehörden empfehlen daher jeder Frau, die eine Schwangerschaft plant, bereits einige Wochen zuvor mit der Einnahme von Folsäure $(0,4$ oder $0,8 \mathrm{mg} /$ Tag) zu beginnen. Frauen, die Antiepileptika einnehmen, empfiehlt man eine höhere Dosis von $5 \mathrm{mg}$ Folsäure pro Tag einzunehmen wegen der folsäureabbauenden Wirkung von Antiepileptika. Allerdings ist nicht bewiesen, dass die Einnahme von Folsäure das Fehlbildungsrisiko des Kindes verringert. Kommt es bei Kindern zu Entwicklungs- oder Intelligenzstörungen, ist eine Valproat-bedingte Störung differenzialdiagnostisch in Betracht zu ziehen. Der Intelligenzquotient der Kinder von Müttern mit niedrig dosierter Valproat-Therapie und gleichzeitiger Gabe von Folsäure war allerdings vergleichbar mit dem der Kinder, deren Mütter andere Antiepileptika eingenommen hatten [10].

Wird Valproat im Kindesalter verordnet, ist mit Eintritt der Menarche die Indikation zur weiteren Behandlung sorgfältig zu überprüfen.

\section{Interessenkonflikt}

$\nabla$

C. E. Elger hat in den letzten zwei Jahren für folgende Firmen bezahlte Vorträge gehalten: Cyberonics, Desitin, Eisai, Medronics, Novartis, UCB. T. Bast gibt Vortrags- und Beratertätigkeit an für Desitin Arzneimittel, UCB, Eisai, Cyberonics und Viropharma. D. Schmidt hat in den letzten zwei Jahren Honorare als Sprecher oder Reisekostenunterstützungen von UCB, Novartis, Eisai, Viropharma und Sun Pharma erhalten.

\section{Literatur}

1 European Medicines Agency. CMDh agrees to strengthen warnings on the use of valproate medicines in women and girls. 21 November 2014: EMA/709243/2014

2 Arzneimittelkommission der deutschen Ärzteschaft. Rote-Hand-Brief zu Arzneimitteln, die Valproat und -verwandte Substanzen enthalten: Risiko für Anomalien des Neugeborenen. (38-2014)

3 Marson AG, Al-Kharusi AM, Alwaidh $M$ et al. The SANAD study of effectiveness of valproate, lamotrigine, or topiramate for generalised and unclassifiable epilepsy: an unblinded randomised controlled trial. Lancet 2007; 369: 1016-1026

4 Christensen J, Grønborg TK, Sørensen MJ et al. Prenatal valproate exposure and risk of autism spectrum disorders and childhood autism. JAMA 2013; 309: 1696-1703

5 Mølgaard-Nielsen D, Hviid A. Newer-generation antiepileptic drugs and the risk of major birth defects. JAMA 2011; 305: 1996-2002

6 Queisser-Luft A, Stolz G, Wiesel A et al. Malformations in newborn: results based on 30,940 infants and fetuses from the Mainz congenital birth defect monitoring system (1990-1998). Arch Gynecol Obstet 2002; 266: $163-167$

7 Morrow J, Russell A, Guthrie E et al. Malformation risks of antiepileptic drugs in pregnancy: a prospective study from the UK Epilepsy and Pregnancy Register. J Neurol Neurosurg Psychiatry 2006; 77: 193-198

8 Campbell E, Kennedy F, Russell A et al. Malformation risks of antiepileptic drug monotherapies in pregnancy: updated results from the UK and Ireland Epilepsy and Pregnancy Registers. J Neurol Neurosurg Psychiatry 2014; 85: 1029-1034

9 Bromley $R$, Weston J, Adab $N$ et al. Treatment for epilepsy in pregnancy: neurodevelopmental outcomes in the child. Cochrane Database Syst Rev 2014: CD010236

10 Meador KJ, Baker GA, Browning $N$ et al. Fetal antiepileptic drug exposure and cognitive outcomes at age 6 years (NEAD study): a prospective observational study. Lancet Neurol 2013; 12: 244-252

11 Bromley RL, Mawer GE, Briggs $M$ et al. The prevalence of neurodevelopmental disorders in children prenatally exposed to antiepileptic drugs. J Neurol Neurosurg Psychiatry 2013; 84: 637-643

12 Diener H, Ackermann H, Hrsg. Leitlinien für Diagnostik und Therapie in der Neurologie: Erster epileptischer Anfall und Epilepsien im Erwachsenenalter. 5. Aufl. Stuttgart: Thieme; 2012: 2-14

13 Schmitz B, Dennig D, Rating $D$ et al. Valproat in der Behandlung epilepsiekranker Frauen. Eine Stellungnahme der Deutschen Gesellschaft für Epileptologie. Nervenarzt 2010; 81: 747 - 752

14 Edey S, Moran N, Nashef L. SUDEP and epilepsy-related mortality in pregnancy. Epilepsia 2014; 4: e72 - e74 